\title{
Desmame difícil e decanulação tardia de pacientes traqueostomizados vítimas de traumas num hospital de referência na Região Norte
}

\author{
Undifficult desmame and late decanntion of tracheostomized trauma victims patients in a referral \\ hospital in the Northern Region
}

\author{
Letícia Barreto Ramos Soares ${ }^{1^{*}} \bullet$, Heloísa Maria Machado e Silva ${ }^{\bullet}$, José Wilson de Araújo \\ Albuquerque Junior ${ }^{30}$
}

\begin{abstract}
${ }^{1}$ Hospital Metropolitano de Urgência e Emergência no Trauma. Ananindeua, Pará, Brasil. ${ }^{2}$ Universidade da Amazônia. Belém, Pará, Brasil. ${ }^{3}$ Hospital Metropolitano de Urgência e Emergência no Trauma. Ananindeua, Pará, Brasil. *Autor para correspondência. E-mail: leticiafono3@hotmail.com
\end{abstract}

\begin{abstract}
Resumo: Fundamentos: A traqueostomia pode ser realizada como ação preventiva, curativa, paliativa ou eletiva, sua indicação muitas vezes acontece devido a falha na extubação, apesar de seus benefícios quando ao conforto respiratório, também há malefícios a longo prazo, no que diz respeito as alterações que refletem diretamente nas funções de deglutição, respiração e fala. Objetivo: Está pesquisa objetivou analisar o processo de desmame difícil e decanulação tardia em pacientes traqueostomizados vítimas de traumas, buscando formas e analisando o que torna um desmame dificil e tardio e como melhorar tal processo. Métodos: Foi realizado um estudo descritivo-análico, retrospectivo e quantitativo, com análise de 40 prontuários, do primeiro semestre de 2018, com pacientes maiores de 18 anos classificados como desmame dificil e decanulação tardia. Tal periodo foi escolhido devido a alta demanda de pacientes com desmame dificil. Para coleta de dados foi utilizado um instrumento contendo informações acerca do diagnóstico funcional e relacionadas ao desmame e decanulação. Resultados: Os pacientes traqeostomizados classificados como desmame tardio em sua maioria são homens, vitimas de acidente motociclistico ou agressões físicas que evoluiram com traumatismo crânio encefálico, e tiveram uma média de 10 a 11 dias de intubação orotraqueal o que foi de grande impacto para o periodo prolongado de traqueostomia. Conclusões: Concluiu-se que os pacientes que tiveram acompanhamento fonoaudiológico desde o periodo de ventilação mecânica invasiva com IOT preste a extubar tiveram menor tempo de desmame da traqueostomia, em comparação com os pacientes que só foram acompanhados após a realização da traqueostomia.
\end{abstract}

Palavras-chave: desmame, fonoaudiologia, traqueostomia.

\begin{abstract}
Background: Tracheostomy can be performed as a preventive, curative, palliative or elective action, ist indication often happens due to failure in extubation, despite its benefits when it comes to respiratory comfort, there are also long-term harms, regarding changes that directly reflect on the swallowing and speech functions. Objective: This research aimed to analyze the process of difficult weaning and late decannulation in tracheostomized patients victims of trauma, looking for ways and analyzing what makes weaning difficult and late and how to improve this process. Methods: A descriptive-analogical, retrospective and quantitative study was conducted, with analysis of 40 medical records, from the first semester of 2018, with patients over 18 years of age classified as difficult weaning and late decannulation. For data collection, an instrument was used containing information about the functional diagnosis and related to weaning and decannulation. Results: Most of the patients undergoing tracheostomy classified as late weaning are men, victims of motorcycle accidents or physical aggressions that evolved with traumatic brain injury, and had an average of 10 to 11 days of orotracheal intubation, which was of great impact for the period prolonged tracheostomy. Conclusions: It was concluded that the patients who had had a speech therapy follow-up since the period of invasive mechanical ventilation with IOT about to extubate had shorter weaning time from the tracheostomy, compared with the patients who were only followed up after the tracheostomy.
\end{abstract}

Keywords: weaning, speech therapy, tracheostomy.

\section{Introdução}

A Traqueostomia (TQT) é um dos procedimentos cirúrgicos mais realizados em todo mundo em pacientes críticos, principalmente quando ocorre a insuficiência respiratória e risco de vida ao indivíduo. Na maioria 
dos casos é realizada ainda no leito da unidade de terapia intensiva (UTI) como forma de diminuir o tempo de intubação orotraqueal (IOT) possibilitando o desmame da sedação e da ventilação mecânica invasiva (VMI) o mais breve possível (Silva, 2020; Pasini et al., 2007; Sakae et al., 2010; Ricz et al., 2011).

Os pacientes vítimas de traumas graves, como traumatismo cranio encefálico, na maioria dos casos, necessitam de traqueostomias de urgência ou eletivas, devido a gravidade e extensão das lesões. Entretando, mesmo trazendo muitos benefícios, a TQT prolongada pode causar disfagias orofaríngeas, dificultando o desmame e retorno alimentar dos pacientes. As alterações da deglutição ocorrem principalmente pela presença do cuff insuflado durante muito tempo, o qual gera restrição na elevação e diminuição da mobilidade laríngea, atrofia da musculatura supra e infra hioidea, ausência de coaptação glótica, devido à falta de fluxo aéreo, e consequente redução da proteção de vias aéreas (Furkim et al.,2014; Garuti et al., 2014; Costa et al., 2016; Medeiros et al; 2019).

Tais malefícios da traqueostomia somado as complicações clínicas advindas do estado clínico grave do paciente, acarretam no aumento do tempo de internação, necessitando de maior aporte de materiais hospitalares (aspirações pulmonares frequentes) e maior sofrimento do paciente (disfunções estomatognáticas - deglutição, fala e respiração), gerando altos custos hospitalares (Furkim et al., 2014).

Para combater tais malefícios e otimizar o desmame da TQT o mais breve possível, se faz necessário a avaliação e acompanhamento fonoaudiológico desde a UTI, buscando assim reabilitar, possibilitando se indicado a decanulação e garantindo a independência funcional ao indivíduo (Zanata et al., 2014). Valendo ressaltar que o processo de decanulação da traqueostomia ocorre por avaliação e acompanhamento de equipe multidisciplinar, composta por fonoaudiólogo, fisioterapeuta, médico e enfermeiro, e a boa interação da equipe é essencial para garantir que o processo seja seguro, em curto prazo, sem complicações ou o menor risco de complicações, evitando falhas e garantindo conforto e bem estar ao paciente (Boulhosa et al., 2015).

Considerando o exposto acima buscou-se apresentar as causas de desmame difícil e decanulação tardia de pacientes vítimas de traumas, assim como apresentar a importância do acompanhamento fonoaudiológico precoce, promovendo o retorno o mais breve possível do indivíduo a sociedade.

\section{Material e métodos}

Estudo realizado no Hospital Metropolitano de Urgência e Emergência no Trauma, localizado na cidade de Ananindeua, Pará - Brasil. Trata-se de um estudo de caráter descritivo-analítico, retrospectivo e quantitativo, a partir da análise de prontuários, sem nenhum tipo de intervenção junto ao paciente.

Foram analizados 40 prontuários de pacientes traqueostomizados, classificados como desmame difícil e decanulação tardia de acordo com o protocolo de desmame de traqueostomia do hospital, ambos os sexos, maiores de 18 anos de idade, atendidos pela equipe de fonoaudiologia no período de janeiro a junho $2018 \mathrm{com}$ diagnósticos de Traumatismo Crânio Encefálico (TCE), Trauma Raquimedular (TRM) ou/e pacientes politraumatizados com TCE ou TRM associado a outras aéreas de trauma.

Foram excluídos da pesquisa prontuários de usuários que não sofreram TCE, nem TRM ou que são classificados como politraumatizados, pacientes que não foram traqueostomizados, idade inferior a 18 anos ou superior a 65 anos, os que tiveram queimadura associada ao trauma, os traqueostomizados que não se enquadravam no processo de desmame e decanulação tardia, ou seja, que realizaram o desmame de traqueostomia de acordo com o protocolo institucional que classifica o desmame normal como o periodo de 24hs com cuff desinsuflado e 48hs com a TQT ocluída e após isso o individuo é decanulado. E também foram excluídos os que por alguma razão não estavam prescritos para acompanhamento fonoaudiológico neste periodo.

Para coleta de dados foi utilizado um instrumento de pesquisa (APÊNDICE A) contendo as seguintes variáveis: registro hospitalar $(\mathrm{RH})$ e número de atendimento, sexo, idade, cidade de origem, mecanismo de trauma, diagnóstico funcional, extensão da lesão, comorbidades associadas, primeiro dia de traqueostomia, data da avaliação fonoaudiológica, data do cuff desinsuflado permanente, oclusão permanente e quantas horas até a decanulação, data da decanulação, tempo de acompanhamento fonoaudiológico até a decanulação, tempo de traqueostomia total, verificar se o paciente foi intubado anteriormente, e o tempo de ventilação mecânica.

Após coletada as informações, foi realizada uma triagem baseada nas dificuldades de manter o cuff desinsuflado por 24 horas, resultado do blue dye test, os que não toleravam a oclusão da traqueostomia por 48 horas e os que evoluiram para traqueostomia metálica. Os pacientes que apresentaram dificudade em 
alguns dos critérios da triagem foram enquadrados como desmame difícil e foram classificados como decanulação tardia.

Depois de eleger os pacientes classificados como desmame difícil e decanulação tardia, a amostra foi dividida em 2 grupos, de acordo com o diagnóstico funcional: Grupo 1 (G1) de TCE e Grupo 2 (G2) de POLITRAUMATIZADOS, TRM não foi incluído nos grupos por que não houve na amostra nenhum caso de diagnostico de TRM isolado, apenas associado, sendo classificado como politraumas.

\section{Resultados e discussão}

A população estudada constituiu-se de 40 (100\%) prontuários de pacientes que se encaixavam no processo de desmame dificil e decanulação tardia de traqueostomia, sendo todos vitimas de traumas, 38 (95\%) eram do sexo masculino e apenas $2(5 \%)$ do sexo feminino. Em relação a faixa etária constatou-se que 19 pacientes tinham entre 18 e 29 anos (47,5\%), 7 pacientes entre os 30 a 40 anos (17,5\%), 11 pacientes de 41 aos 59 anos (27,5\%) e 3 pacientes entre 60 e 65 anos $(7,5 \%)$.

Os homens expõem-se mais aos fatores de risco, seja no que se refere ao sedentarismo, tabagismo, obesidade, alcoolismo, ou relativos ao ambiente, possivelmente, pelos padrões de educação para o sexo masculino e a manifestação da agressividade em comparação com os padrões femininos. A problemática da morbimortalidade por causas externas, é considerada como a primeira causa de morte entre homens em idade produtiva, sendo elas, principalmente as agressões e os acidentes de transportes, que geram um grande problema de saúde pública, devido o aumento dos custos sociais e econômicos necessários ao tratamento e reabilitação dessas vítimas (Tavares et al., 2014). Como evidenciado no estudo que $95 \%$ da amostra são homens em idade produtiva, demonstrando o que vários autores ja descreveram em outros estudos que os jovens adultos tem maior prevalência e exposição a acidentes de trânsito, queda e agressões físicas (Ott et al., 1993; Tavares et al., 2014; Rodrigues et al., 2017; Moreira et al., 2018).

Em relação aos mecanismos de trauma encontrados durante a coleta de dados, evidenciou-se que $40 \%$ dos pacientes sofreram acidente motociclistico. Somando aos acidentes motociclisticos, os $12,5 \%$ dos atropelamentos e 2,5\% dos acidentes de carro, temos $55 \%$ de acidentes relacionados ao trânsito.

Os acidentes de trânsito, pricipalmente os motociclisticos, tornaram-se um grande problema de saúde pública, tendo em vista que no estudo atual $40 \%$ da amostra teve como mecanismo de trauma o acidente de moto. Um estudo mostrou que foram realizadas 145.920 internações financiadas pelo SUS, gerando cerca de 187 milhões de custos públicos (Silva et al., 2015). Em outro estudo constatou-se que de 2008 a 2010 ocorreu um aumento de $7 \%$ nos óbitos por transportes terrestres e $23 \%$ foram por acidentes de motocicletas. Tento o TCE como principal determinante de óbito e sequelas em vítimas politraumatizadas (Albuquerque et al., 2016).

Os mecanismos de trauma desencadearam lesões graves dividas em 2 grupos: G1 - Traumatismo Crânio Encefálico (TCE) isolado (30 - 75\%) e G2 - Politraumatizados (10 - 25\%). Sendo que dentro dos Politraumatizados houveram subdivisões de acordo com as lesões associadas que são: TCE e trauma de face (7 - 17,5\%); TCE, TRM e trauma de face (2 - 5\%); TRM e trauma de face (1 - 2,5\%). Não aprensentando nenhum paciente com TRM isolado.

No Brasil e em outros paises as lesões traumáticas são as principais causas de mortes de pessoas dos 5 aos 44 anos e correspondem a $10 \%$ do total de mortes. Gerando danos socioeconômicos enormes. O Trauma craniano é responsável por $75 \%$ a $97 \%$ das mortes por traumas em crianças e jovens e as ocorrências aumentam a cada ano, como evidenciado neste estudo que $75 \%$ dos traumas foi o TCE isolado e mais $22,5 \%$ dos politraumatizados também tinham associado o TCE (Albuquerque et al., 2016).

Tais traumas graves levaram a necessidade de suporte avançado de vida, e todos os pacientes da amostra foram intubados e colocados em ventilação mecânica invasiva antes de serem traqueostomizados, sendo analizado o tempo de tubo orotraqueal (TOT) em dias, tendo como achado que os pacientes passaram de 8 a 16 dias intubados, no G1 a maior incidência de dias foi entre 10 e 11 dias para 14 pacientes (46,6\%), e no G2 foi de 8 a 9 dias para 5 pacientes (50\%).

Em relação ao tempo de intubação orotraqueal tivemos um intervalo de 8 a 16 dias de TOT, tendo maior prevalência 10 a 11 dias de TOT para 14 pacientes do grupo 1 (G1) com 46,6\% da amostra e de 8 a 9 dias de TOT para 5 pacientes do grupo 2 (G2) com $50 \%$ da mostra. Na literatura o tempo máximo de dias de permanência do TOT e o momento ideal para realização da TQT ainda é alvo de muita discurssão e controvérsia, principalmente por ser uma necessidade individual em que não é possível prever o tempo necessário de ventilação mecânica, sendo assim sua indicação é feita por critérios subjetivos e funcional levando em consideração as particularidades de cada paciente. Porém há estudos que sinalizam que no $7^{\circ}$ dia 
de TOT já é indicado a TQT, por ser o momento em que as lesões traqueais começam a aparecer (Cardoso et al., 2014). Neste estudo tivemos um número de dias satisfatórios e bem próximos aos indicados na literatura.

A partir da realização da TQT iniciou-se o desmame da ventilação mecânica para a partir daí iniciar o desmame da própria traqueostomia. Os pacientes que foram caracterizados como desmame difícil com decanulação tardia, tiveram entre 10 a 88 dias até a decanulação, sendo que no G1 intervalo maior foi de 10 a 20 dias para 10 pacientes (33,3\%), e no G2 foi de 21 a 30 dias para 4 pacientes (40\%).

Dentre as dificuldades do desmame da TQT o que mais se destacou foi que dos 40 estudados, 33 deles conseguiram manter a TQT ocluída por 24 horas após tolerar o cuff desinsuflado, mas ultrapassaram as 48 horas para decanulação por apresentarem complicações, tais como, processos infecciosos advindos de infecções hospitalares, por aguardo de cirúrgias e piora no padrão respiratório.

Na literatura não foi encontrado referências acusando o tempo máximo de uso da traqueostomia, isso irá depender da necessidade e dificuldades de cada paciente. No presente estudo destacou-se que no G1 33,3\% da amostra levou de 10 a 20 dias até a decanulação e no G2 tivemos que $40 \%$ da amostra levou de 21 a 30 dias até ser decanulado, como no G2 temos associação de traumas e com isso maior número de cirurgias e complicações, justifica-se o maior tempo de traqueostomia em comparação com os que tiveram apenas TCE.

Em contrapartida quando separamos a amostra total dos pacientes em: os que foram acompanhados pela equipe de fonoaudiologia desde a intubação ( 23 pacientes), destes 23 pacientes tiveram tempo médio de TQT de 22,6 dias e os que só foram acompanhados após a realização da traqueostomia (17 pacientes), destes 17 pacientes tiveram tempo médio de TQT de 39,2 dias.

Observa-se que atuação da fonoaudiologia de forma preventiva desde o periodo de intubação Orotraqueal destes individuos trás dados importantes acerca do menor tempo de desmame de traqueostomia. Na literatura ainda não há estudos relacionados a está atuação, somente recomenda-se a atuação fonoaudiológica após 24hs a $48 \mathrm{hs}$ pós extubação. Porém, neste estudo observa-se que o gerenciamento fonoaudiológico após a indicação da equipe multidisciplicar para programação de extubação e a atuação da fonoaudiologia neste processo antes da traqueostomia foi de grande relevância para o desmame mais rápido de TQT.

\section{Conclusão}

Conclui-se que é fundamental o acompanhamento fonoaudiológico no paciente crítico vítima de trauma de forma precoce e preventiva, garantindo um atendimento multidisciplinar desde a unidade de terapia intensiva, levando em consideração as complicações clinicas e buscando formas de minimizar tais complicações junto a equipe assistencial.

As infecções hospitalares são frequentes em pacientes de longa permanência, e refletindo em cima disso é que destaco a importância de seguir os cuidados e higienização dos profissionais de saúde, pois, no estudo observamos que uma das justificativas para o processo de tardio de decanulação foram relacionadas a piora infeciosa do paciente.

Destacamos também a importância do acompanhamento fonoaudiológico intensivo, para otimizar o diagnóstico precoce de complicações relacionadas a deglutição, fala, voz e motricidade orofacial, atuando de forma indireta e direta nesses casos como medida de prevenção e segurança para uma extubação e ou posterior processo de desmame de traqueostomia seguro e com menor risco de falha, garantindo o retorno do indivíduo a sociedade com qualidade de vida e em menor tempo de internação hospitalar.

Tal pesquisa trás dados novos e que ainda necessitam de muito estudo científico e técnico.

\section{Agradecimento}

Agradecemos aos profissionais que ousaram ir além das suas dúvidas, medos e opressões e que constantemente trazem novidades e novos aprendizados a Fonoaudiologia.

\section{Referências}

Albuquerque, A. M. , Silva, H. C. L., Torquato, I. M. B., Gouveia, B. L. A., Abrantes, M. S. A. P., \& Torres, V. S. F. 2016. Vítimas de acidentes de moto com traumatismo. Revista de Enfermagem UFPE, 10(5), 17301738.

Boulhosa, F. J. S., Costa, L. R. N., Lima, G. M., de Paula Gonçalves, K. L., Amaral, J. A. R., Silva, P. K. E., ... \& Feio, S. D. C. A. 2015. O Impacto do protocolo de desmame de traqueostomia em pacientes vítimas de Traumatismo Cranioencefálico internados no Hospital Metropolitano de Urgência e Emergência no Pará. Revista da Universidade Vale do Rio Verde, 13(2), 313-323. 
Cardoso, L., Simoneti, F. S., Camacho, E. C., Lucena, R. V., Guerra, A. F., \& da Silva Rodrigues, J. M. (2014). Intubação orotraqueal prolongada e a indicação de traqueostomia. Revista da Faculdade de Ciências Médicas de Sorocaba, 16(4), 170-173.

Costa, C. C., Favero, T. C., da Rosa, F. B., dos Santos Steidl, E. M., \& Mancopes, R. (2016). Decanulação: atuação fonoaudiológica e fisioterapêutica. Distúrbios da Comunicação, 28(1), 92-101.

Furkim, M., \& Rodrigues, K. 2014. Disfagias nas unidades de terapia intensiva. São Paulo, SP: Rocca.

Garuti, G., Reverberi, C., Briganti, A., Massobrio, M., Lombardi, F., \& Lusuardi, M. 2014. Swallowing disorders in tracheostomised patients: a multidisciplinary/multiprofessional approach in decannulation protocols. Multidisciplinary respiratory medicine, 9(1), 1-10.

Medeiros, G. C. D., Sassi, F. C., Lirani-Silva, C., \& Andrade, C. R. F. D. 2019. Critérios para decanulação da traqueostomia: revisão de literatura. In CoDAS (vol. 31, No. 6), Sociedade Brasileira de Fonoaudiologia.

Moreira, M. R., Ribeiro, J. M., Motta, C. T., \& Motta, J. I. J. 2018. Mortalidade por acidentes de transporte de trânsito em adolescentes e jovens, Brasil, 1996-2015: cumpriremos o ODS 3.6?. Ciência \& Saúde Coletiva, 23, 2785-2796.

Ott, E. A., Favaretto, A. L., R Neto, A. F., Zechin, J. G., \& Bordin, R. 1993. Acidentes de trânsito em área metropolitana da região sul do Brasil: caracterização da vítima e das lesões. Revista de Saúde Pública, 27, 350-356.

Pasini, R. L., Fernandes, Y. B., Araújo, S., \& Soares, S. M. D. T. P. 2007. A influência da traqueostomia precoce no desmame ventilatório de pacientes com traumatismo craniencefálico grave. Revista Brasileira de Terapia Intensiva, 19(2), 176-181.

Ricz, H. M. A., de Mello Filho, F. V., de Freitas, L. C. C., \& Mamede, R. C. C. M. 2011. Traqueostomia. Medicina (Ribeirão Preto), 44(1), 63-69.

Rodrigues, C. L., Rizzo, T. S. A., Merici, G., Ribas, M., \& Górios, C. 2017. Perfil epidemiológico dos acidentes de trânsito entre crianças e adolescentes. Arquivos Catarinenses de Medicina, 46(4), 91-102.

Sakae, T. M., Henrique, B. C., Prates, M. A. S., Maraschin, J. F., \& Silva, R. M. 2010. Traqueostomia precoce e tardia em pacientes de uma unidade de terapia intensiva no sul do Brasil. Revista da Sociedade Brasileira de Clínica Médica, 8, 500-504.

Silva, F. D., Boes, A. A., Lazzari, D. D., Busana, J. D. A., Nascimento, E. R. P. D., \& Jung, W. (2015). Vítimas de trauma por acidente de moto atendidas em serviço móvel de urgência. Revista de Enfermagem. UFPI, 71-78.

Silva, I. L. D. 2020. Impacto da traqueostomia no tempo de ventilação mecânica invasiva em pacientes adultos: revisão narrativa.

Tavares, F. L., Coelho, M. J., \& Leite, F. M. C. (2014). Homens e acidentes motociclísticos: caracterização dos acidentes a partir do atendimento pré-hospitalar. Escola Anna Nery, 18(4), 656-661.

Zanata, I. D. L., Santos, R. S., \& Hirata, G. C. (2014). Tracheal decannulation protocol in patients affected by traumatic brain injury. International Archives of Otorhinolaryngology, 18(2), 108-114.

\section{Minicurrículo}

Letícia Barreto Ramos Soares. Fonoaudióloga formada pela Universidade da Amazônia (UNAMA), Esp. em Urgência e Emergência no Trauma (UEPA), Esp. em Linguagem e Aprendizagem (INCISA), Mestranda em Saúde Pública (UNEATLANTICO E UNINI), pós-graduanda de Fonoaudiologia Intensiva e Disfagia pela Finama, com formação em Eletroestimulação aplicada à fonoaudiologia pela CPÓS (Centro Educacional), Método FonoTaping Elástica pela FIAMA e Laserterapia para fonoaudiólogos por Sávio Bastos e Atualmente é diretora e responsável técnica da empresa Fono Intensiva, fonoaudióloga do Hospital Metropolitano de Urgência e Emergência no Trauma e Hospital Adventista de Belém, prioritariamente atuando nas Unidades de terapia intensiva adulto, Covid-19, neonatologia e pediatria e exerce o cargo de Preceptora do Programa de Residência Multiprofissional em urgência e emergência no Trauma no Hospital Metropolitano.

Heloísa Maria Machado e Silva. Possui graduação em Fonoaudiologia pelo Instituto Superior de Estudos Edy Pinheiros (1978), especialização em linguagem humana pela Universidade da Amazonia, Mestre em Desenvolvimento e Meio Ambiente Urbano pela Universidade da Amazônia. 
José Wilson de Araújo Albuquerque Junior. Fonoaudiólogo formado pela Universidade da Amazônia (UNAMA), especializado em fonoaudiologia hospitalar pela CPÓS, com formação em Eletroestimulação aplicada à fonoaudiologia pela CPÓS, Método FonoTaping Elástica pela FINAMA e Laserterapia para fonoaudiólogos por Sávio Bastos e Atualmente é pós-graduando de Fonoaudiologia Intensiva e Disfagia pela FINAMA, diretor e responsável técnico da empresa Fono Intensiva, fonoaudiólogo do Hospital Metropolitano de Urgência e Emergência no Trauma, Hospital Adventista de Belém e Hospital Geral da Unimed e exerce o cargo de Preceptor do Programa de Residência Multiprofissional em urgência e emergência no Trauma no Hospital Metropolitano.

Como citar: Soares, L.B.R., Machado e Silva, H.M., \& Albuquerque Junior, J.W.A. 2021. Desmame difícil e decanulação tardia de pacientes traqueostomizados vítimas de traumas num hospital de referência na Região Norte. Pubsaúde, 5, 125. DOI: https://dx.doi.org/10.31533/pubsaude5.a125

Recebido: 6 jan. 2020.

Revisado e aceito: 18 jan. 2021.

Conflito de interesse: os autores declaram, em relação aos produtos e companhias descritos nesse artigo, não ter interesses associativos, comerciais, de propriedade ou financeiros que representem conflito de interesse.

Licenciamento: Este artigo é publicado na modalidade Acesso Aberto sob a licença Creative Commons Atribuição 4.0 (CC-BY 4.0). 